Vol. 40 (1989) [363-364]

\title{
ON A PROBLEM OF SZÁSZ
}

\author{
Yasuyuki Hirano
}

\section{Dedicated to Professor Miyuki Yamada on his 60th birthday.}

\begin{abstract}
Let $R$ be a ring with centre $Z$. In this note, we prove the following: If the additive group $Z^{+}$of $Z$ has finite group-theoretic index in $R^{+}$, then $R$ has an ideal $I$ contained in $Z$ such that $R / I$ is a finite ring. This is a solution of a problem posed by F.A. Szász.
\end{abstract}

Throughout this note, $R$ denotes a ring with centre $Z$. We write $R^{+}$for the additive group of $R$, and $C(R)$ for the commutator ideal of $R$.

In Problem 84 of [1], F.A. Szász asks: In which rings $R$ has the additive group $Z^{+}$of the centre $Z ;$ a finite group-theoretic index in $R^{+}$? We show that such a ring $R$ has an ideal $I$ contained in $Z$ such that $R / I$ is a finite ring.

We begin with the following

Proposition 1. If $Z^{+}$has finite index in $R^{+}$, then $C(R)$ is finite.

Proof: Let $n$ be the index of $Z^{+}$in $R^{+}$and $\left\{r_{1}=0, r_{2}, \ldots, r_{n}\right\}$ a complete set of coset representatives of $Z^{+}$in $R^{+}$. Since $\left[r_{i}+z, r_{j}+z^{\prime}\right]=\left[r_{i}, r_{j}\right], C(R)$ is additively generated by $r\left[r_{i}, r_{j}\right] s$ where $r, s \in R$. But $r\left[r_{i}, r_{j}\right]=\left(r_{k}+z\right)\left[r_{i}, r_{j}\right]=$ $r_{k}\left[r_{i}, r_{j}\right]+\left[r_{i} z, r_{j}\right]$ and $\left[r_{i} z, r_{j}\right]=\left[r_{l}+z^{\prime}, r_{j}\right]=\left[r_{l}, r_{j}\right]$. A similar result holds for $\left[r_{i}, r_{j}\right] s$ and so $C(R)$ is additively generated by the finite set $\left\{r_{k}\left[r_{i}, r_{j}\right],\left[r_{i}, r_{j}\right] r_{l}, r_{k}\left[r_{i}, r_{j}\right] r_{l} \mid 2 \leqslant\right.$ $i, j, k \leqslant n\}$. Also each $\left[r_{i}, r_{j}\right]$ has finite additive order, otherwise there exist distinct integers $n$ and $n^{\prime}$ such that $\left(n-n^{\prime}\right)\left[r_{i}, r_{j}\right] \neq 0$ but $n r_{i}+Z^{+}=n^{\prime} r_{i}+Z^{+}$. However the latter equation yields $\left(n-n^{\prime}\right) r_{i} \in Z$, so $0=\left[\left(n-n^{\prime}\right) r_{i}, r_{j}\right]=\left(n-n^{\prime}\right)\left[r_{i}, r_{j}\right]$, a contradiction. Hence, as an abelian group, $C(R)$ has a finite set of generators of finite order, and so is finite.

Proposition 2. Assume that $C(R)$ is finite. Then there exists a finite nilpotent ideal $N$ of $R$ such that $R / N$ is the direct sum of a funite semisimple ring and a commutative ring.

Proof: Let $N$ be the Jacobian radical of $C(R)$. It is easily seen that $N$ is a finite nilpotent ideal of $R$. Let $R^{\prime}=R / N$. Since $C\left(R^{\prime}\right)$ is the canonical homorphic image

Received 9 November, 1988

Copyright Clearance Centre, Inc. Serial-fee code: 0004-9729/89 \$A2.00+0.00. 
of $C(R)$ in $R / N, C\left(R^{\prime}\right)$ is a finite semisimple ring. Hence $C\left(R^{\prime}\right)$ has an identity $e$. Since $C\left(R^{\prime}\right)$ is an ideal of $R^{\prime}$, e is a central idempotent of $R^{\prime}$. Hence $R^{\prime}=C\left(R^{\prime}\right) \oplus S$, where $S=\{r-r e \mid r \in R\}$. Clearly, $S$ is a commutative ring.

As an immediate consequence of Propositions 1 and 2, we have

Corollary 1. Let $R$ be a semiprime ring with centre $Z$. Then the following statements are equivalent:

(1) $Z^{+}$has finite index in $R^{+}$;

(2) $C(R)$ is finite;

(3) $R$ is the direct sum of a finite ring and a commutative ring.

The following example shows that the statements (1) and (2) are not equivalent in general.

Example. Let $\mathbb{Z}$ denote the ring of integers. Let $R$ be the set of all matrices $\left(\begin{array}{ll}a & b \\ 0 & c\end{array}\right)$ with $a, c \in \mathbb{Z}$ and $b \in \mathbb{Z} / 2 \mathbb{Z}$. In $R$, define addition and multiplication as in ordinary matrices. Then $R$ is a ring. The ideal $I=\left(\begin{array}{cc}2 Z & 0 \\ 0 & 2 Z\end{array}\right)$ is contained in the centre of $Z$ and $R$, and $R / I$ is a finite ring. Hence $Z^{+}$has finite index in $R^{+}$. However $R$ does not satisify (3) in Corollary 1.

Now we come to our main theorem.

ThEOREM 1. Let $R$ be a ring with centre $Z$. Then the following statements are equivalent:

(1) The additive group $Z^{+}$of $Z$ has finite index in $R^{+}$;

(2) $R$ has an ideal $I$ contained in $Z$ such that $R / I$ is a finite ring.

Proof: It suffices to prove the implication (1) $\Rightarrow(2)$. By Proposition $1, C(R)$ is a finite ideal. Let $f: Z \rightarrow \operatorname{End}(C(R))$ be the ring homomorphism defined by $f(z)(r)=r z$ for all $z \in Z$ and $r \in C(R)$, and let $I=\operatorname{Ker} f$. Then $Z / I$ is a finite ring. Let $r_{R}(C(R))$ denote the right annihilator of $C(R)$ in $R$. Then, $I=r_{R}(C(R)) \cap Z$. Let $a \in I$ and $x \in R$. Then, for any $y$ in $R$, we get $[a x, y]=a[x, y]=0$. Hence, $a x \in Z$, and so $a x \in r_{R}(C(R)) \cap Z=I$. This proves that $I$ is an ideal of $R$. Since $Z / I$ is finite and since $Z^{+}$has finite index in $R^{+}, R / I$ is a finite ring. This completes the proof.

\section{REFERENCES}

[1] F.A. Szász, Radicals of Rings (John Wiley and Sons, Chichester, New York, Brisbane, Toronto, 1981). 